Laser Chem. 1983, Vol. 3, pp. 279-292

0278-6273/83/0306-0279 $\$ 12.00 / 0$

(C) harwood academic publishers gmbh

Printed in Great Britain

\title{
Studies of Molecular Adsorbates at Interfaces by Optical Second-Harmonic Generation $\dagger$
}

\author{
H. W. K. TOM, T. F. HEINZ and Y. R. SHEN
}

Department of Physics, University of California, Berkeley, California 94720, and Materials and Molecular Research Division, Lawrence Berkeley Laboratory, Berkeley, California 94720

Optical second-harmonic generation (SHG) is surface-specific and has the sensitivity to detect submonolayers of molecules. It can be applied to study molecular adsorbates at the interface between any two centrosymmetric media. This has been demonstrated by using dye and $p$-nitrobenzoic acid (PNBA) molecules adsorbed on smooth fused quartz substrates as examples. With resonant SHG, the $S_{0} \rightarrow S_{2}$ transition of the adsorbed dye molecules was measured. From the polarization dependence of the SHG, the orientation of PNBA at both the air/quartz and the ethanol/quartz interfaces was deduced. By varying the concentration of PNBA in ethanol, an adsorption isotherm for PNBA at the ethanol/quartz interfaces was also obtained.

Laser applications to surface science have received little attention in the past. Recently, however, a number of new discoveries have excited increasing interest in this area. Through the processes of resonant fluorescence and multiphoton ionization, lasers have been used to study molecules scattered from a surface, ${ }^{1}$ thermally desorbed from a surface, and catalytically produced from species adsorbed on a surface. ${ }^{2}$ Through excitation of molecules, they can also serve to induce molecular desorption, ${ }^{3}$ to change the molecule-surface interaction, and to modify catalytical reactions. ${ }^{2}$ Then, with lasers as the excitation source, several new surface probes have been developed.

$\dagger$ This work was supported by the Director, Office of Energy Research, Office of Basic Energy Sciences, Materials Sciences Division of the US Department of Energy under Contract Number DE-ACO3-76SF00098. 
These include laser-induced desorption, ${ }^{3}$ surface photoacoustic spectroscopy, ${ }^{4}$ and the nonlinear optical techniques of Raman gain spectroscopy ${ }^{5}$ surface coherent anti-Stokes-Raman scattering, ${ }^{6}$ and surface second-harmonic generation. ${ }^{7}$ This paper will review the present status of our development of second-harmonic generation (SHG) as a probe of molecular adsorbates.

Nonlinear optical techniques in general are attractive because they afford spectral resolution limited only by the laser linewidth and time resolution (for dynamic studies) in the picosecond or subpicosecond domain. As we shall see later, SHG, in particular, is appropriate for in-situ investigation of the interface between any two centrosymmetric media (provided one of them is transparent) and for measurement of the orientation of molecular adsorbates at such an interface. These are characteristics not commonly possessed by the conventional surface probes. They make SHG a potential useful technique for surface studies.

Any effective surface probe must discriminate against the bulk media to discern the weaker surface signal. This is usually achieved by spectral discrimination. For the scattering of low-energy electrons, it is the limited penetration depth of the electrons into the bulk which gives rise to the surface sensitivity. In the case of SHG, however, it is the difference of structural symmetries between the surface and the bulk that allows the discrimination, although resonant SHG can also spectrally distinguish the surface from the bulk.

As an introduction to SHG, one can refer to the following simple picture. Consider an incoming laser beam with frequency $\omega$ propagating in a medium composed of a collection of molecules. Through the linear and nonlinear polarizabilities of the molecules, the laser field induces on each molecule a dipole oscillating not only at frequency $\omega$, but also at the harmonic frequencies. This system of oscillating dipoles then acts as an antenna array radiating coherently at both the fundamental and the harmonic frequencies. For SHG, we can usually write the induced dipole moment on the $i$ th molecule as $\mathbf{p}_{i}^{(2)}(2 \omega)=$ $\boldsymbol{\alpha}_{i}^{(2)}: \mathbf{E}(\omega) \mathbf{E}(\omega)$, with a corresponding nonlinear polarization of

$$
\mathbf{P}^{(2)}(2 \omega)=\sum_{i=1}^{N} \mathbf{p}_{i}^{(2)}(2 \omega)=\chi^{(2)}: \mathbf{E}(\omega) \mathbf{E}(\omega)
$$

for the system. In these expressions $\boldsymbol{\alpha}_{i}^{(2)}$ is the second-order polarizability, $\chi^{(2)}=\sum_{i=1}^{N} \alpha_{i}^{(2)}$ is the second-order susceptibility, and $N$ is the 
number of molecules per unit volume. The resulting electric field at the second harmonic will then be proportional to $\mathbf{P}^{(2)}(2 \omega)$. From symmetry considerations, it is clear that for centrosymmetric molecules, $\boldsymbol{\alpha}^{(2)}$ vanishes in the electric-dipole approximation. Similarly, $\boldsymbol{x}^{(2)}$ vanishes if the system as a whole has inversion symmetry. Thus, SHG is only allowed in a medium where non-centrosymmetric molecules are arranged in a non-centrosymmetric way.

In a medium with inversion symmetry, the magnetic-dipole contribution to the nonlinear polarization could still lead to SHG in the bulk, but it is generally very weak. ${ }^{8}$ At an interface, however, the surface atomic or molecular layer is always non-centrosymmetric. Therefore, SHG from the surface layer is certainly allowed. Its strength could be larger than or at least comparable with that from the bulk. By the same token, SHG from adsorbed molecules is also allowed. In many cases, the SH signal from the adsorbed species can actually dominate over that from the substrate. We can then use SHG as an effective probe to study the adsorbed species. ${ }^{7}$

The second-order nonlinearity of the adsorbed molecular layer can arise (a) from an ordered orientation of asymmetric molecules $\left(\alpha^{(2)} \neq\right.$ $0)$ and (b) from the molecule-substrate interaction. The latter generally consists of two parts: the electromagnetic interaction, which can be expressed in terms of a microscopic local-field correction, and the chemical interaction, which modifies the electronic charge distribution around the adsorbed molecules. These interactions lead to an effective $\boldsymbol{\alpha}^{(2)}$ for the adsorbed molecules. Even if the molecules are centrosymmetric, the interactions will break the inversion symmetry and yield a nonvanishing effective $\boldsymbol{\alpha}^{(2)}$. While the modification of $\boldsymbol{\alpha}^{(2)}$ by the molecule-substrate interaction may be important in general, in this paper we shall consider only the case where the intrinsic second-order nonlinearity of the molecules dominates.

We can make a crude estimate of the second-order nonlinearity of an asymmetric molecule using a simplified expression derived from the full second-order perturbation calculation: ${ }^{8}$

$$
\left|\alpha^{(2)}\right| \sim \frac{e^{3}}{\hbar^{2}} \frac{\left\langle r^{3}\right\rangle}{\omega_{0}^{2}}
$$

Here $\langle r\rangle$ is effective radius of the molecule and $\omega_{0}$ is the average transition frequency. With $\langle r\rangle \sim\left\langle r^{3}\right\rangle^{1 / 3} \sim 2-5 \AA$ and $\hbar \omega_{0} \sim 10^{-11} \mathrm{erg}$, we find $\left|\alpha^{(2)}\right| \sim 5 \times 10^{-30}-10^{-28}$ esu. If the molecules are perfectly 
aligned to form a monolayer, this would yield a surface nonlinear susceptibility $\left|\chi_{s}^{(2)}\right|=N_{s}\left|\alpha^{(2)}\right| \sim 10^{-15}-2 \times 10^{-14} \mathrm{esu}$, where we have taken the surface density of molecules to be $N_{S}=2 \times 10^{14} \mathrm{~cm}^{2}$.

To estimate the SH output from such a layer of molecules, we refer to the expression of Bloembergen and Pershan ${ }^{9}$, for SHG from a thin film. We can then write a reduced formula for the $\mathrm{SH}$ radiation as

$$
S(2 \omega) \simeq \frac{2^{10} \pi^{3} \omega}{\hbar c^{3}\left|\varepsilon^{4}(\omega) \varepsilon^{2}(2 \omega)\right|}\left|\chi_{s}^{(2)}\right|^{2} I^{2}(\omega) A T \quad \frac{\text { photons }}{\text { pulse }}
$$

assuming that the adsorbed molecules form a layer on a substrate of dielectric constant $\varepsilon$, the laser intensity $I(\omega)$ is in ergs $/ \mathrm{cm}^{2}$, the beam area $A$ is in $\mathrm{cm}^{2}$, and the laser pulse duration $T$ is in sec. Here the angular and polarization dependences have been omitted. For $\left|\chi \chi_{s}^{(2)}\right| \sim$ $10^{-15}$ esu, $I(\omega) \sim 10 \mathrm{MW} / \mathrm{cm}^{2}$ at $1.06 \mu \mathrm{m}, A \sim 1 \mathrm{~cm}^{2}, T \sim 10 \mathrm{~ns}$, and $\varepsilon \sim 2.5$, we find $S(2 \omega) \sim 10^{3}$ photons/pulse, which should be readily detectable. For molecules adsorbed on a smooth substrate, the output is coherent and highly directional, as dictated by the boundary conditions. This facilitates detection of the signal because the coherent phase of the output can also be used to discriminate against the background contribution from the substrate, and the high directionality of the output allows spatial filtering to be used to suppress luminescence and scattered radiation.

In the estimate above, we have neglected the molecule-substrate interaction and a possible macroscopic local-field correction. The former could lead to a larger effective $\left|\alpha^{(2)}\right|$, while the latter could increase the macroscopic field actually seen by the molecules. In fact, it has been shown that on a rough silver surface, the macroscopic local field can be enhanced by more than an order of magnitude through the local plasmon resonances and the lightning rod effect. ${ }^{10}$ As a result, the surface SHG can be enhanced by as much as four orders of magnitude. ${ }^{11}$ This makes SHG from molecules adsorbed on a rough $\mathrm{Ag}$ surface easily observable despite the fact that the output is scattered in all directions. For example, even with an input as weak as $0.2 \mathrm{~mJ} / 02 . \mathrm{cm}^{2}$ in a $10-\mathrm{ns}$ pulse, the observed $\mathrm{SH}$ output from a monolayer of pyridine on rough $\mathrm{Ag}$ can be $\sim 10^{6}$ photons/pulse. ${ }^{12}$

An attractive feature SHG as a surface probe is the simplicity of the experimental setup. As shown in Figure 1, all that is required is 


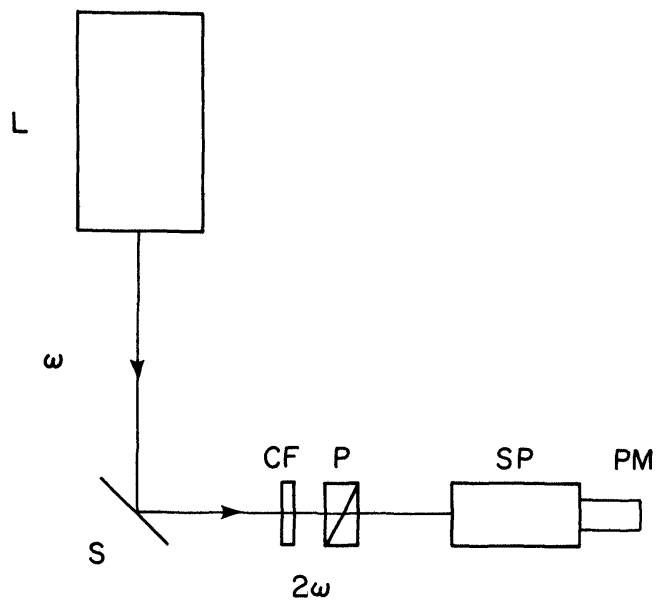

XBL8212-7389

FIGURE 1 Schematic representation of the experimental setup for a measurement of surface SHG. The radiation at the harmonic frequency $2 \omega$ generated by the sample is isolated by filtering elements and detected with a photomultiplier.

a medium-power pulsed laser and a detection system with appropriate collection optics and filtering elements. For spectroscopic studies, a tunable laser is needed. Either the forward or backward SHG can be detected, as best suits the actual experimental situation.

To illustrate that SHG can be used for spectroscopic studies of submonolayers of adsorbates, we have investigated rhodamine dyes adsorbed on fused quartz. ${ }^{7}$ Figure 2 a shows that the $S_{0} \rightarrow S_{2}$ electronic transition of the dye molecules can be reached by a two-photon excitation with a dye laser. Resonant SHG from the molecules is then possible because the molecules are non-centrosymmetric. By scanning the laser frequency around the $S_{0} \rightarrow S_{2}$ transition, a resonant peak should be observed in the SH output versus frequency. The samples of dye molecules adsorbed on smooth fused quartz plates were prepared by the spinning technique. From the measured optical density of the strong $S_{0} \rightarrow S_{1}$ transition for a stack of samples, an average surface coverage of $\sim 5 \times 10^{13}$ dye molecules $/ \mathrm{cm}^{2}$ was deduced, which corresponds to roughly a half monolayer. In our experiment, dye laser pulses of $1 \mathrm{~mJ}$ energy, $10 \mathrm{~ns}$ duration, and $1 \mathrm{~cm}^{-1}$ linewidth were focused to an area of $\sim 10^{-3} \mathrm{~cm}^{2}$ on the sample. The reflected 


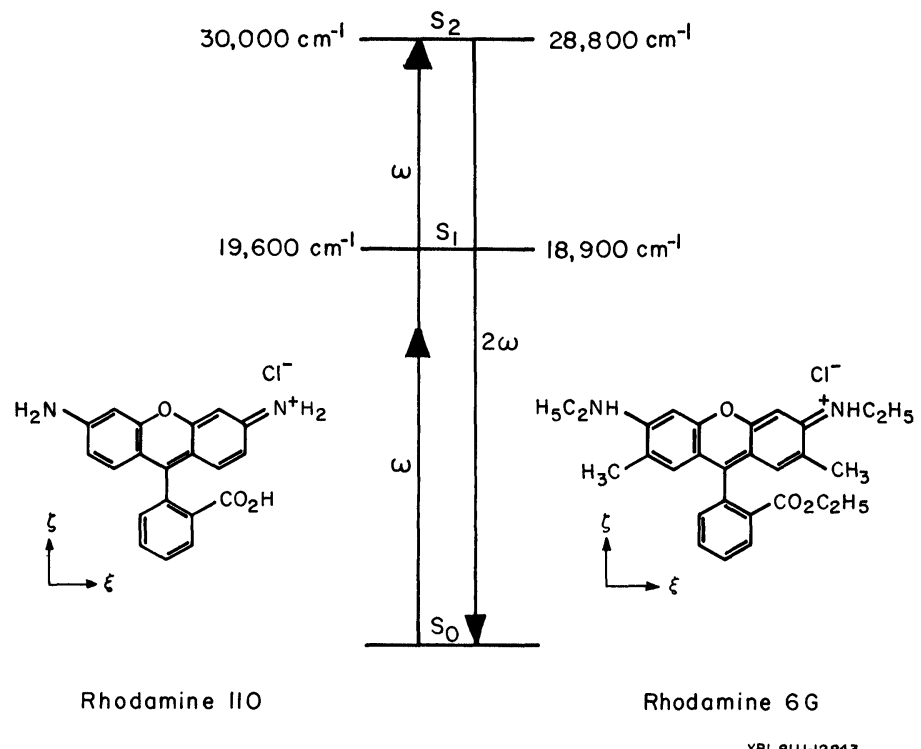

XBL $8111-12843$

FIGURE 2 Resonant SHG in rhodamine 110 and rhodamine 6G. (a) indicates the structures and energy levels of the two dyes, as well as the resonant SHG process. (b) shows the experimental results for surface SHG by the dye molecules adsorbed on fused quartz at submonolayer coverages. The normalized second-harmonic intensity is given as a function of the second-harmonic wavelength in the region of the $S_{0} \rightarrow S_{2}$ transition.

SH output was found to consist of $\sim 10^{4}$ photons/pulse at the resonant peak. The $\mathrm{SH}$ output as a function of the $\mathrm{SH}$ wavelength is shown in Figure 2b. Two kinds of dyes, rhodamine 6G and rhodamine 110, were studied. Their molecular structures are different only in their substituents, as is reflected in the relatively small shifts of their energy levels. In Figure 2b, the resonant SH peaks at the $S_{0} \rightarrow S_{2}$ transition frequencies for the two dyes are clearly resolved. The signal here was a few orders of magnitude higher than the background SHG from the bare quartz plate. This indicates that SHG should have the sensitivity to measure the spectrum of much less than $\frac{1}{10}$ of a monolayer of dye molecules.

While SHG can be a sensitive probe of surface spectroscopy, some sophisticated linear optical techniques may prove to be equally sensitive. The real advantage of surface SH spectroscopy actually lies in 


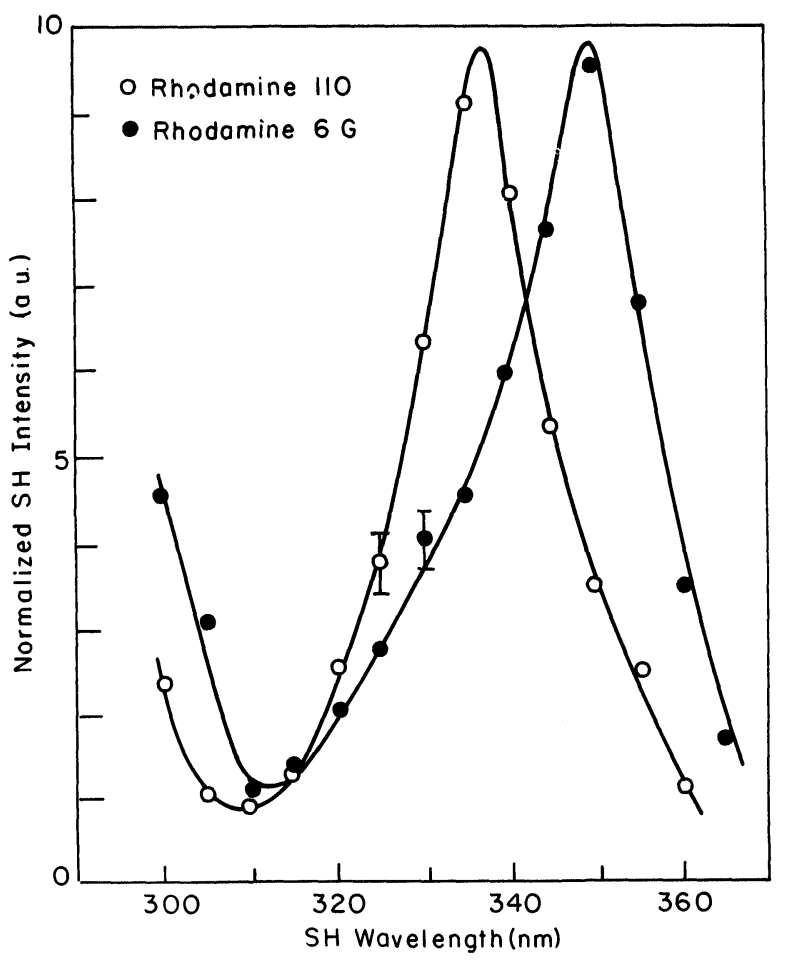

XBL 8110-6773

FIGURE 2(b).

its capacity for transient spectroscopic studies of surfaces. Since ultrashort laser pulses can be used for SHG (with, in fact, the advantage of having a higher output resulting from the higher peak laser power), a time resolution of better than a picosecond should be possible. Transient surface spectroscopy with a submonolayer detection sensitivity and a subpicosecond time resolution would certainly constitute a very exciting field of research.

Surface SHG can also be used to deduce information about the orientation of the molecular adsorbates. The basic idea is quite simple. The polarization properties of the SHG should depend on the tensorial character of $\chi_{s}^{(2)}$ and on the average molecular orientation. Thus, in those cases where one has sufficient knowledge about $\alpha^{(2)}$, one should 
be able to obtain the average molecular orientation from the polarization measurements of SHG. More explicitly, we have $\chi_{S}^{(2)}$ related to $\boldsymbol{\alpha}^{(2)}$ by

$$
\begin{aligned}
\left(\chi^{(2)}\right)_{i j k} & =\sum_{n=1}^{N}\left(\alpha_{n}^{(2)}\right)_{i j k} \\
& =N\left\langle G_{i j k}^{\xi \eta \xi}(\theta, \phi, \psi)\right\rangle \alpha_{\xi \eta \zeta}^{(2)}
\end{aligned}
$$

where $\left\langle G_{i j k}^{\xi \eta \xi}(\theta, \phi, \psi)\right\rangle$ is the average coordinate transformation factor connecting the lab coordinates $(i, j, k)$ to the coordinates $(\xi, \eta, \zeta)$ attached to the molecules, and $\theta, \phi$, and $\psi$ are the Euler angles describing the orientation of the molecules. Knowing $\left(\chi_{s}^{(2)}\right)_{i j k}$ and $\alpha_{\xi \eta \xi}^{(2)}$, one can find $\left\langle G_{i j k}^{\xi \eta \xi}(\theta, \phi, \psi)\right\rangle$ and, hence, some information about the average molecular orientation.

The form of $\chi_{s}^{(2)}$ itself also reflects the symmetry of the twodimensional arrangement of the adsorbed molecules. For example, for a distribution isotropic with respect to directions in the plane, the only independent nonvanishing elements of $\chi_{s}^{(2)}$ are $\left(\chi_{s}^{(2)}\right)_{z i i}$ and $\left(\chi_{s}^{(2)}\right)_{i z i}=(\chi s)_{i i z}^{(2)}$, with $i=x, y$, or $z$. The SH output should remain unchanged when the sample is rotated about its surface normal, irrespective of the incident angle and polarization.

In the case of rhodamine dye molecules on fused quartz, we indeed found results consistent with an isotropic distribution of molecules on the surface. The polarization dependence suggested that the molecules did not lie flat on the surface, but had an orientation in which the molecular plane was perpendicular to the surface and the nearly symmetric axis $\hat{\zeta}$ was tilted from the surface normal by $\sim 34^{\circ}$.

In surface science, one would generally like to deal with structures less complex than the dye molecules. In order to show that surface SHG can also be applied to smaller adsorbates, while still avoiding the use of a high vacuum system for sample preparation, we have chosen p-nitrobenzoic acid (PNBA) on fused quartz as another test case. As indicated in Figure 3, the PNBA molecule has a fairly simple structure. According to a theoretical estimate, ${ }^{13} \alpha^{(2)}$ of the molecule is dominated by the axial component $\alpha_{\zeta \zeta \zeta}^{(2)}$, with $\hat{\zeta}$ along the principal molecular axis; the other more important nonvanishing elements $\alpha_{\xi \xi \xi}^{(2)}=\alpha_{\xi \zeta \xi}^{(2)}$ and $\alpha_{\xi \xi \xi}^{(2)}$ (with $\hat{\xi}$ in the molecular plane) are about one order of magnitude smaller than $\alpha_{\xi \zeta \zeta}^{(2)}$. As a first-order approximation, we can therefore assume $\alpha_{\zeta \zeta \zeta}^{(2)}$ is the only nonvanishing element of 
<smiles>O=C(O)c1ccc([N+](=O)[O-])cc1</smiles>

(a)

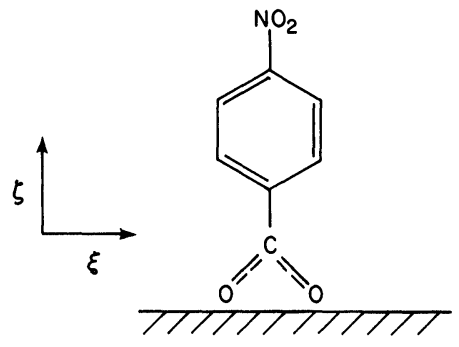

(b)

$X B L 8212-7390$

FIGURE 3 Molecular structure of $p$-nitrobenzoic acid (PNBA). (a) describes the free molecule and (b) the chemisorbed species.

$\boldsymbol{\alpha}^{(2)}$. This simplification is equivalent to the assumption that the molecule can be treated as a long rod. The orientation of the molecules on a surface is then described by the single tilt angle $\theta$ that the molecular $\zeta$ axis makes with the surface normal. For an isotropic surface distribution of PNBA, the nonvanishing elements of $\chi_{s}^{(2)}$ are now related to $\alpha_{\zeta 6 \zeta}^{(2)}$ via $\theta$ by the following equations:

$$
\begin{aligned}
& \left(\chi_{s}^{(2)}\right)_{z z z}=N_{S} \alpha_{\zeta \zeta \zeta}^{(2)}\left\langle\cos ^{3} \theta\right\rangle \\
& \left(\chi_{S}^{(2)}\right)_{z i i}=\left(\chi_{S}^{(2)}\right)_{i z i}=\frac{1}{2} N_{S} \alpha_{\zeta \zeta \zeta}^{(2)}\left\langle\sin ^{2} \theta \cos \theta\right\rangle
\end{aligned}
$$

where $i=x, y$ is a direction in the plane of the substrate and $z$ is the direction of the surface normal. The factor $\frac{1}{2}$ arises from averaging over the azimuthal angle for the isotropic distribution. The above equation leads to the ratio

$$
R \equiv \frac{\left(\chi_{s}^{(2)}\right)_{z z z}+2\left(\chi_{s}^{(2)}\right)_{z x x}}{\left(\chi_{s}^{(2)}\right)_{x z x}}=\frac{2\langle\cos \theta\rangle}{\left\langle\sin ^{2} \theta \cos \theta\right\rangle}
$$

If we make the simplifying assumption that all molecules have the same tilt angle, then the above ratio becomes

$$
R=2 / \sin ^{2} \theta
$$

By measuring $R$, thne angle $\theta$ is determined.

PNBA is believed to be chemisorbed on fused quartz through the symmetric structure of the ionized acid group. ${ }^{14}$ This modification, however, should not change $\alpha^{(2)}$ of the molecule very much because 
the major contribution to $\boldsymbol{\alpha}^{(2)}$ comes from the distorted $\pi$-electron system of the benzene ring. Consequently, it is reasonable to assume that the adsorbed PNBA molecules still preserve the symmetry that $\boldsymbol{\alpha}^{(2)}$ is dominated by $\alpha_{\xi \zeta \zeta}^{(2)}$.

To find the ratio $R$ in Eq. (5), it is generally not advisable to measure the different components of $\chi_{S}^{(2)}$ separately, as laser fluctuations are likely to induce inaccuracies. This is particularly true when the surface SH output is very weak, in which case even the direct determination of $R$ from the ratio of the simultaneously measured components of $\chi_{s}^{(2)}$ by the same laser would not be very accurate. We present here a simple scheme which allows us to infer $R$ from a single polarization measurement, namely, from the null position of an output analyzer.

Let the incoming laser field be $\mathbf{E}(\omega)$. The $\mathrm{SH}$ output from the adsorbed molecules will have $s$ - and $p$-polarized components given respectively by

$$
\begin{aligned}
& E_{p}(2 \omega)=\left[A\left(\chi_{s}^{(2)}\right)_{z z z}+B\left(\chi_{s}^{(2)}\right)_{z x x}+C\left(\chi_{s}^{(2)}\right)_{x z x}\right] E^{2}(\omega) \\
& E_{s}(2 \omega)=D\left(\chi_{s}^{(2)}\right)_{x z x} E^{2}(\omega)
\end{aligned}
$$

where $A, B, C$, and $D$ are coefficients depending on the incident angle and polarization of the laser beam. If an analyzer is inserted in the output path with its polarizing axis rotated by an angle $\phi$ away from the direction of $s$ polarization, the $\mathrm{SH}$ field after the analyzer becomes

$$
\begin{aligned}
E(2 \omega) & =E_{s}(2 \omega) \cos \phi+E_{p}(2 \omega) \sin \phi \\
& =A \sin \phi\left[\left(\chi_{S}^{(2)}\right)_{z z z}+b\left(\chi_{S}^{(2)}\right)_{z x x}-f\left(x_{S}^{(2)}\right)_{x z x}\right] E^{2}(\omega)
\end{aligned}
$$

where $b=B / A$ and $f=-(C+D \cot \phi) / A$, both of which depend on the input polarization and geometry. We can, for example, set $b=2$ by varying the input polarization for a given input geometry. If we then rotate the analyzer to $\phi=\phi_{0}$ to null the output, we find, from $E(2 \omega)=0$ in Eq. (8), that $f$ becomes identical to $R$ in Eq. (5). Since $f$ is known from the given input polarization and geometry and the measured nulling angle $\phi_{0}$, the orientational angle $\theta$ is given by Eq. (6). The method described here has the advantage of not relying on any intensity measurement, but only on finding the polarization null angle. In general, the $\mathrm{SH}$ output may be elliptically polarized, in which case a polarizer-compensator set should be used as the analyzer. 
We have applied the above technique to the measurement of the orientation of PNBA on a smooth fused quartz plate. A submonolayer of PNBA was prepared by placing a known volume of a dilute solution of PNBA on the substrate and allowing the solution to evaporate. In order to demonstrate that the surface SHG technique is applicable to the study of adsorbed molecules not only at air/solid interfaces, but also at interfaces between two condensed media, we have measured the orientation of PNBA adsorbed on fused quartz both in air and in ethanol solution. The results are indicated in Table I. Three different laser frequencies were used in the measurement of PNBA at the ethanol/fused quartz interface. The $0.532-\mu \mathrm{m}$ excitation is in two-photon resonance with the first significant electronic transition of PNBA. A strong dispersion is therefore expected in the SHG from PNBA. However, if our technique of measuring the orientation of PNBA is indeed valid, the measured value of $\theta$ should certainly be independent of the exciting laser frequency. As seen in Table I, we have found that this is the case within our experimental accuracy. The average orientation angle of PNBA at the ethanol/fused quartz interface was measured to be $\theta=39 \pm 3^{\circ}$. The accuracy could probably be improved by careful control of the stress-induced birefringence observed in our substrate. The same technique for PNBA adsorbed at an air/fused quartz interface yielded $\theta=70 \pm 3^{\circ}$. That the adsorbed molecules are tilted more towards the surface normal in liquid than in air is probably due to the dielectric effect of the liquid medium.

In the above discussion, we have neglected the effect of local fields on the determination of molecular orientation. The local fields here arise from both the molecule-molecule interaction and the

TABLE 1

Data for surface SHG by PNBA adsorbed on a fused quartz substrate. Experimental values for the ratio $R$ and the corresponding average adsorbate orientation are listed for laser excitation at the three indicated pump wavelengths

\begin{tabular}{ccc}
\hline$\lambda$ & $R=\frac{\left(\chi_{s}^{(2)}\right)_{z z z}+2\left(\chi_{S}^{(2)}\right)_{z x x}}{\left(\chi_{s}^{(2)}\right)_{x z x}}$ & $\theta$ \\
\hline $1.06 \mu \mathrm{m}$ & $5.3 \pm 0.5$ & $38 \pm 3^{\circ}$ \\
$683 \mathrm{~nm}$ & $4.4 \pm 0.5$ & $42 \pm 3^{\circ}$ \\
$532 \mathrm{~nm}$ & $5.5 \pm 0.5$ & $37 \pm 3^{\circ}$ \\
\hline
\end{tabular}


molecule-substrate interaction. With the local-field correction, the relation between $\chi_{S}^{(2)}$ and $\alpha^{(2)}$ becomes

$$
\left(\chi_{S}^{(2)}\right)_{i j k}=N\left\langle\boldsymbol{\alpha}_{i j k}^{(2)} L_{i}(2 \omega) L_{j}(\omega) L_{k}(\omega)\right\rangle
$$

where the $L$ 's (assumed diagonal) are the local-field correction factors. Since $L_{i}(2 \omega) \neq L_{j}(\omega) \neq L_{k}(\omega)$, ignoring these factors could lead to an erroneous determination of the molecular orientation. To see whether or not the local-field correction is important, we can use a point dipole model to evaluate the $L$ 's. ${ }^{15}$ The molecule-molecule interaction is described by the induced dipole-induced dipole interaction and the molecule-substrate by the dipole-image dipole interaction. It is found ${ }^{16}$ that for $N_{S}=2 \times 10^{14}$ molecules $/ \mathrm{cm}^{2}$ and $\alpha^{(1)}=10 \AA^{3}$, the local-field correction factors are $L_{x}=L_{y} \sim 1$, and $L_{z}=\left(1+\frac{1}{4} \cos ^{2} \theta\right)^{-1}$, which is also not very different from 1 . Thus, in the case of PNBA on fused quartz, the local field should not be important in the determination of the molecular orientation. This conclusion is also supported by the experimental fact that the measured orientation angle $\theta$ was independent of the exciting laser frequency. It is expected that the local-field correction should have a strong dispersion near resonance. If the effect of local fields were important in the determination of $\theta$, the results obtained with different laser frequencies would certainly fail to agree.

With the capability of probing adsorbates at a liquid/solid interface, we can measure the adsorption isotherm, i.e., the surface coverage of adsorbed molecules in equilibrium with the molecules in solution. We have made such a measurement for PNBA at the ethanol/fused quartz interface. The results are given in Figure 4. By fitting the data with a simple Langmuir equation for adsorption, we can deduce the change of free energy in adsorption of PNBA on fused quartz from ethanol to be $\sim 8 \mathrm{kcal} / \mathrm{mol}$.

In summary, we have shown that SHG can indeed be a potentially useful probe for surface studies, in particular, for the investigation of molecular adsorption at a variety of different interfaces. Several important aspects of the technique are not yet well understood. For example, how does the substrate contribution to SHG limit the sensitivity of the technique and how will the local-field correction affect the results in the case of high surface densities of small molecules adsorbed on metals or on semiconductors. In surface science, the vibrational spectroscopy of molecular adsorbates plays a most impor- 


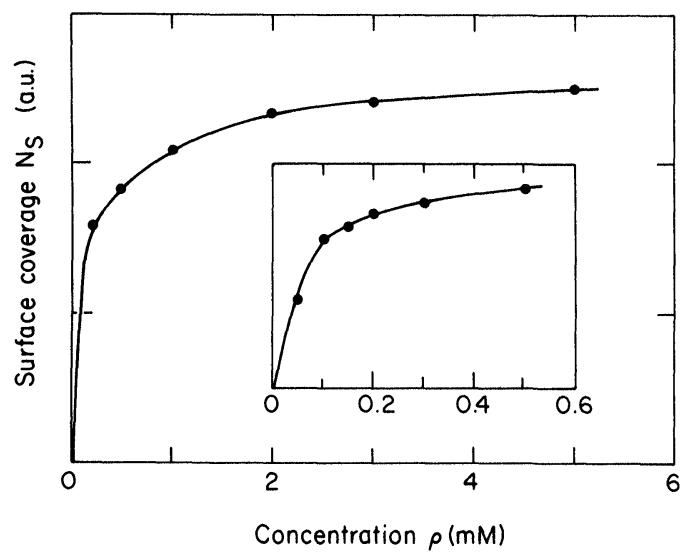

FIGURE 4 Isotherm for the adsorption of PNBA on fused quartz out of an ethanolic solution. The behavior for lower concentrations of dissolved PNBA is shown in the inset. The data were obtained by means of surface SHG with an $s$-polarized pump at $532 \mathrm{~nm}$.

tant role. Such information can be obtained by means of resonant sum-frequency generation, $\omega=\omega_{1}+\omega_{2}$, with $\omega_{1}$ tunable over the vibrational excitations. Again, this nonlinear optical technique has the advantages of being able to study adsorbates at interfaces between any two centrosymmetric media (one of them being transparent), to obtain spectra of adsorbates with very high spectral resolution, to investigate the dynamic properties of adsorbates with a time resolution better than a picosecond, and to determine, with the help of selective excitation of a vibrational mode, the orientation of adsorbates. The requirement of a tunable infrared laser could impede the advance of the technique, but the problem may be solved by the development of infrared free electron lasers.

\section{References}

1. See, for example, M. Asscher, W. L. Guthrie, T. H. Lin and G. A. Somorjai, Phys. Rev. Lett. 49, 76 (1982).

2. See, for example, M. C. Lin, XIth International Quantum Electronics Conference, Boston (June 1980), paper D.6.

3. T. J. Chuang and H. Seki, Phys. Rev. Lett. 49, 382 (1982).

4. F. Träger, H. Coufal and T. J. Chuang, Phys. Rev. Lett. 49, 1720 (1982). 
5. J. P. Heritage and D. L. Allara, Chem. Phys. Lett. 74, 507 (1980).

6. C. K. Chen, A. R. B. de Castro, Y. R. Shen and F. DeMartini, Phys. Rev. Lett. 43, 946 (1979).

7. T. F. Heinz, C. K. Chen, D. Ricard and Y. R. Shen, Phys. Rev. Lett. 48, 478 (1982).

8. See, for example, N. Bloembergen, Nonlinear Optics (Benjamin, New York, 1977).

9. N. Bloembergen and P. S. Pershan, Phys. Rev. 128, 606 (1962).

10. J. Gersten and A. Nitzan, J. Chem. Phys. 73, 3023 (1980).

11. C. K. Chen, A. R. B. de Castro and Y. R. Shen, Phys. Rev. Lett. 46, 145 (1981).

12. C. K. Chen, T. F. Heinz, D. Ricard and Y. R. Shen, Phys. Rev. Lett. 46, 1010 (1981).

13. Following S. J. Lalama and A. F. Garito, Phys. Rev. A 20, 1179 (1979).

14. J. L. Hall and P. K. Hansma, Surf Sci. 76, 61 (1978) and references therein.

15. A. Bagchi, R. G. Barrera and B. B. Dasgupta, Phys. Rev. Lett. 44, 1475 (1980);

A. Bagchi, R. G. Barrera and R. Fuchs, Phys. Rev. B 25, 7086 (1982).

16. P. Ye (unpublished). 